\title{
The Effects of Early Removal of Indwelling Urinary Catheter after Radical Hysterectomy ${ }^{1}$
}

\author{
Donald H. Chamberlain, M.D. ${ }^{*, 2}$ Michael P. Hopkins, M.D., ${ }^{*}$ James A. Roberts, M.D., ${ }^{*}$ Edward J. \\ McGuire, M.D. $\dagger$ George W. Morley, M.D., ${ }^{*}$ and Chichung Wang, M.D. $\dagger$ \\ ${ }^{*}$ Department of Obstetrics and Gynecology, Division of Gynecologic Oncology, and $\dagger$ Department of Surgery, Section of Urology, University of \\ Michigan Medical Center, Ann Arbor, Michigan 48109-0718
}

Received October 26, 1990

\begin{abstract}
Radical hysterectomy has long been a primary mode of therapy for selected gynecologic malignancies. The lower urinary tract is an area associated with complications following this procedure. Lack of satisfactory reflex micturition and urinary retention, diminished bladder sensation, infection, and fistula formation are common adverse sequelae. Prolonged indwelling catheterization is a cornerstone of postoperative management after radical hysterectomy. An alternative regimen consisting of early postoperative catheter removal, with a strict voiding schedule, and intermittent self-catheterization (ISC) for postvoid residuals (PVR) was prospectively investigated. Intermittent self-catheterization was initiated only if the PVR $12 \mathrm{hr}$ after catheter removal was greater than $75 \mathrm{ml}$. Twenty-six patients who underwent radical hysterectomy were studied. Catheters were removed between the fifth and ninth postoperative day. Eighteen patients $(69 \%)$ had PVRs less than $75 \mathrm{ml}$ at $12 \mathrm{hr}$ and were successfully managed with a strict voiding schedule only. Eight patients (31\%) had 12hr PVRs greater than $75 \mathrm{ml}$ and were managed with a strict voiding schedule and ISC until the PVR was less than $75 \mathrm{ml}$ for two consecutive voids. These patients were evaluated with fluorourodynamics and none had an abnormal study. Compared to 25 historical control patients, study group median indwelling catheter duration was less (6.0 days compared to 30.0 days) with no increase in postoperative complications. On the basis of these data, early removal of indwelling urinary catheters after radical hysterectomy appears to be an acceptable alternative to long-term catheterization. 1991 Academic Press, Inc.
\end{abstract}

\section{INTRODUCTION}

Radical hysterectomy with pelvic lymphadenectomy continues to play a significant role in the treatment of

\footnotetext{
1 The opinions and assertions contained herein are those of the authors and are not to be construed as official or as representing the views of the Department of the Air Force or the Department of Defense.

${ }^{2}$ To whom reprint requests should be addressed at Department of OB/GYN, SGHSO, Keesler USAF Medical Center, Keesler AFB, MS 39534.
}

selected gynecologic malignancies. As a result of the extensive dissection, lower urinary tract dysfunction is the most common complication associated with radical hysterectomy [1-3]. Urinary retention, detrusor hypertonicity, hypotonicity, areflexia, diminished vesical sensation, infection, stress incontinence, and fistula formation remain the most frequently reported postoperative complications [3-7]. Improved surgical technique, use of perioperative antibiotic prophylaxis, drainage of the retroperitoneal space, and prolonged urinary catheterization are believed to be responsible for the decreased rate of serious urinary tract complications. For example, fistula formation has declined from a reported rate of greater than $10 \%$ in 1962 [2] to current rates of less than $2 \%$ [8]. Although vesical abnormalities as assessed by cystometrics, urethral pressure profiles, and EMG studies have been well documented [5-10], the contribution of prolonged indwelling catheterization to bladder dysfunction is unclear.

This study was undertaken to evaluate the feasibility, complications, and effects on voiding of early catheter removal after radical hysterectomy.

\section{METHODS AND MATERIALS}

Patients undergoing radical hysterectomy at the University of Michigan Medical Center between February 1, 1989 , and January 31 , 1990 , were prospectively managed with a protocol consisting of short-term urinary catheterization followed by placement on a voiding schedule and intermittent self-catheterization (ISC) for measurement of postvoid residuals (PVR) on the basis of their postvoid residual $12 \mathrm{hr}$ after catheter removal. Patients were assessed preoperatively with a complete genitourinary history, including prior GU surgery, stress incontinence, frequent urinary tract infections (greater than two 
per year), or prior bladder dysfunction. A preoperative postvoid residual was obtained for all patients. Complete preoperative cystometrics were not performed on a routine basis as the purpose of the study was to assess voiding patterns and bladder residuals after radical surgery. When the history was unremarkable it was assumed that preoperative cystometrics would not provide further information relevant to postoperative voiding patterns. $\mathrm{Pa}$ tients were treated with a type III radical hysterectomy [11], including upper vaginectomy, pelvic lymphadenectomy, and complete resection of the cardinal and uterosacral ligaments. Indwelling, transurethral, Foley catheters were placed in the operating room and were removed on Postoperative Day 5 or 6, depending on the availability of "next-day" fluorourodynamics. Removal beyond this period was dependent on the occurrence of nongenitourinary complications.

Patients were placed on a strict voiding schedule consisting of voids at 4-hr intervals, or more frequently if the urge to void was noted. They were counseled to anticipate decreased bladder sensation with difficulty in initiating micturition. Patients were instructed to utilize perineal relaxation with valsalva to aid in voiding.

After each void, patients were instructed in ISC and used this technique to measure the PVR. Twelve hours after the removal of the indwelling catheter each patient was asked to void and the PVR was measured. If the PVR was greater than $75 \mathrm{ml}$ each patient was instructed to continue the strict voiding schedule with ISC for PVR until her postvoid residual was less than $75 \mathrm{ml}$ for two consecutive voids. Patients were instructed to continue on the voiding schedule until their postoperative clinic appointment at 6 weeks. Patients with residuals greater than $75 \mathrm{ml}$ at $12 \mathrm{hr}$ were assessed fluorourodynamicly (FUDS) prior to discharge from the hospital and monthly thereafter if PVRs remained greater than $75 \mathrm{ml}$. Patients with 12-hr PVRs less than $75 \mathrm{ml}$ were managed with the strict voiding schedule only. At regular intervals (monthly for 3 months then every 3 months for 9 months) all paticnts were assessed for decreased bladder sensation, difficulty voiding, incontinence, infection, or other genitourinary complaint.

The study population was compared to 25 historical control patients. The control group consisted of 25 consecutive radical hysterectomy cases performed at the University of Michigan Medical Center immediately preceding the start of the study. The control population was compared to the study group with respect to age, height, weight, estrogen status, body surface area, estimated blood loss, duration of radical surgery, postoperative complications, daily narcotic usage (in parenteral morphine sulfate equivalents), extent of radical surgery, indwelling catheter duration, and postoperative bladder dysfunction.
TABLE 1

\begin{tabular}{lccc}
\hline & $\begin{array}{c}\text { Study } \\
\text { patients } \\
(n=26)\end{array}$ & $\begin{array}{c}\text { Historical } \\
\text { control } \\
\text { patients } \\
(n=25)\end{array}$ & \\
\hline Age & 40.2 & 36.4 & NS \\
BSA & 1.75 & 1.72 & NS \\
EBL & 1139 & 1146 & NS \\
Duration of & 4.4 & 4.6 & NS \\
$\quad$ surgery & 34.2 & 28.2 & NS \\
Narcotic usage & 4.0 & 4.0 & NS \\
Bowel function & & &
\end{tabular}

Note. Age in years; BSA, body surface area in M2; EBL, estimated blood loss in ml; duration of surgery, mean hours; narcotic usage, mean per day of morphine sulfate equivalents; bowel function, mean return on postoperative day.

\section{RESULTS}

Thirty-two patients were treated with radical hysterectomy and pelvic lymphadenectomy. Patients with intraoperative lower urinary tract injury, prior genitourinary surgery for other than the correction of stress incontinence, or inability to perform ISC were ineligible for enrollment. Twenty-six patients were eligible and were managed with this protocol. Six patients were ineligible on the basis of intraoperative lower urinary tract injury (four patients) or inability to perform ISC (two patients). Age, estrogen status, body surface area, postoperative narcotic usage, estimated blood loss, duration of surgery, extent of surgery, and time for return of bowel function were not statistically different from those of control patients (Table 1). Tumor histologies were similar in both groups. All study patients were managed with transurethral Foley catheters while 23 of 25 control patients $(92 \%)$ were managed with suprapubic catheterization (per the usual practice of the attending gynecologic oncologist).

Suprapubic catheters were placed through a small (2$\mathrm{cm}$ ) vesical incision and exited through a separate skin incision. Placement of suprapubic catheters did not increase the duration or extent of surgery significantly when compared to that of study patients. Catheters were removed in 4 to 6 weeks at the discretion of the attending physician.

In the study group indwelling Foley catheters were removed, on average, the sixth postoperative day (median, 6 days; range, 5-9 days). Twenty-two of twenty-six catheters $(85 \%)$ were removed on the fifth or sixth postoperative day, depending on the availability of next-day fluorourodynamics. The remaining catheters were removed later due to nongenitourinary complications [prolonged ileus (2), cuff cellulitis (1), and postoperative cisplatin-based chemotherapy (1)]. The control group was catheterized for an average of 29 days (median, 30 days; 


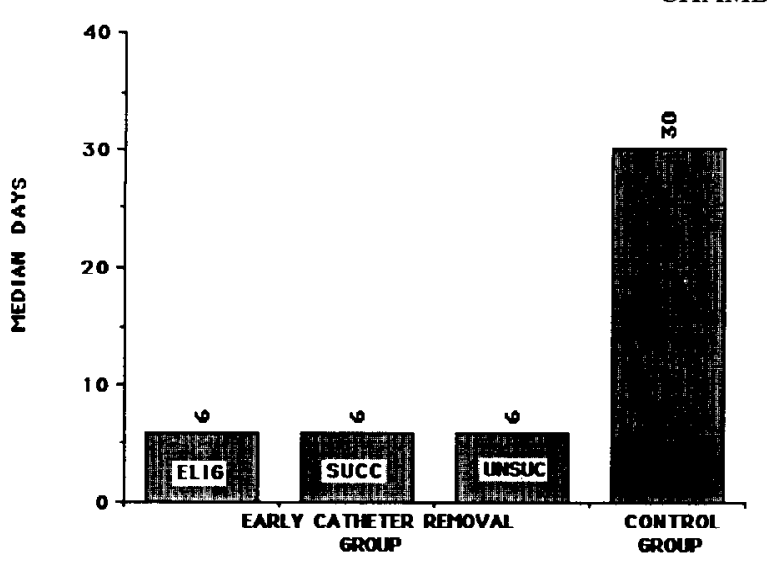

FIG. 1. Postoperative day urinary catheter removed in median days. Early catheter removal group: ELIG, all patients treated with early catheter removal; SUCC, PVR $<75 \mathrm{ml}$ at $12 \mathrm{hr}$; UNSUC, PVR $>75$ ml at 12 hr. Control group: historical control patients.

range, 8-44 days) (Fig. 1). Minor urinary tract complications such as subclinical stress incontinence (not requiring the usage of a protective pad and no interference with daily activity) or decreased bladder sensation were not significantly different between the study and historical control groups. These sequelae occurred in less than $20 \%$ of patients without regard to modality of postoperative catheter management. Clinically significant infections (cystitis or suprapubic exit-site cellulitis) occurred more frequently in the control group than in the study group [four episodes verses one (not statistically significant)].

Prolonged catheterization, defined as indwelling catheterization for greater than 30 days after surgery in historical control patients and ISC for PVR for greater than 30 days after surgery in study patients, occurred significantly more often in the control group than in study patients $[P<0.01 ; 11(44 \%)$ vs $3(11.5 \%)]$ (Fig. 2). There were no serious catheter-associated complications in either group.

Within the study group, 18 patients $(69 \%)$ had postvoid

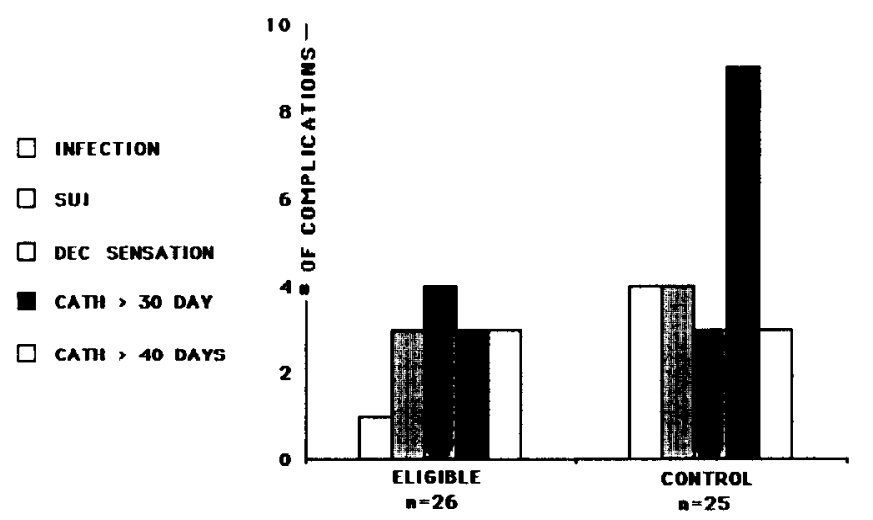

FIG. 2. Number of minor postoperative genitourinary complications.

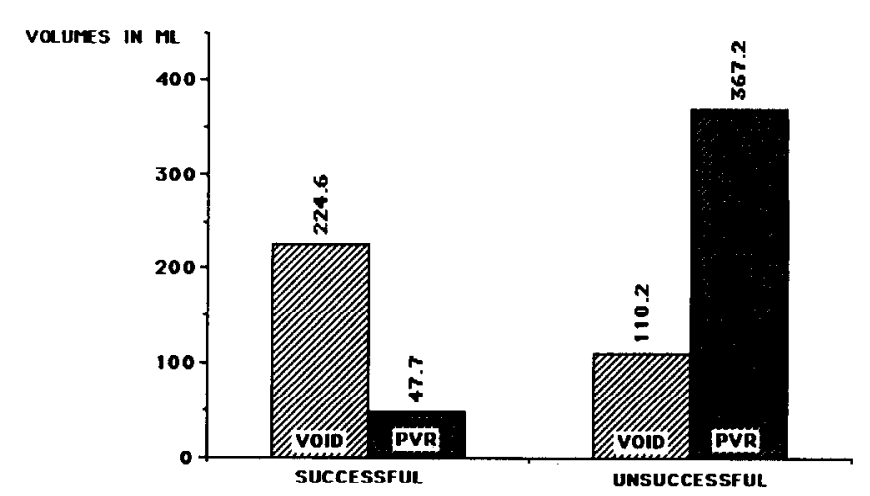

FIG. 3. Mean void volume and PVR for $12 \mathrm{hr}$ following removal of indwelling catheters in paticnts managed with voiding schedule only (PVR $<75 \mathrm{ml}$ at $12 \mathrm{hr}$ ) verses those in patients managed with continued ISC (PVR $>75 \mathrm{ml}$ at $12 \mathrm{hr}$ ).

residuals $12 \mathrm{hr}$ after removal of catheters of less than 75 $\mathrm{ml}$ (mean, $40.6 \mathrm{ml}$; range, $1-75 \mathrm{ml}$ ). These patients were managed with the strict voiding schedule only. There were no episodes of urinary retention, fistulae, or serious infections in this group. Eight patients $(31 \%)$ required continued ISC for postvoid residuals greater than $75 \mathrm{ml} 12$ hr after removal of their catheters.

In the eight patients who required continued ISC, the median duration of ISC before their PVRs were less than $75 \mathrm{ml}$ on two consecutive voids was 19.5 days (range, 7165 days). Three of the eight patients required ISC for PVR in excess of 30 days (56, 109, and 165 days).

Seven of the eight patients $(88 \%)$ who had PVRs at $12 \mathrm{hr}$ of greater than $75 \mathrm{ml}$ underwent video fluorourodynamic assessment within $24 \mathrm{hr}$ after catheter removal (one patient was discharged prior to FUDS). These patients all showed decreased sensation, normal vesical compliance, and normal proximal urethral sphincter function. No patient showed evidence of increased resting or filling pressures or abnormal reflex vesical contractility. All of these patients were either unable to void or had high residual volumes at the completion of FUDS. No fistulae or serious infections occurred in these patients. When compared to patients not requiring ISC, these patients had smaller void volumes (mean, $110.2 \mathrm{ml}$ vs $224.6 \mathrm{ml}$ ) and larger PVRs $(P=0.02$; mean, $367.5 \mathrm{ml}$ vs $47.5 \mathrm{ml})$ (Fig. 3). Patients requiring prolonged ISC after catheter removal did not differ significantly in age, body surface area, estimated blood loss, duration of surgery, extent of surgery, average postoperative narcotic usage, return of bowel function, or postoperative day of catheter removal (Table 2).

\section{DISCUSSION}

Lower urinary tract dysfunction remains the most commonly encountered adverse sequela following radical hys- 
TABLE 2

\begin{tabular}{lccc}
\hline & $\begin{array}{c}\text { Void schedule } \\
\text { only patients }\end{array}$ & $\begin{array}{c}\text { ISC for PVR } \\
\text { patients }\end{array}$ & \\
\hline Age & 41.8 & 36.6 & NS \\
BSA & 1.74 & 1.78 & NS \\
EBL & 1231 & 931 & NS \\
Duration of & 4.3 & 4.6 & NS \\
$\quad$ surgery & 33.3 & & NS \\
Narcotic usage & 4.3 & 36.1 & NS \\
Bowel function & 6.0 & 3.4 & NS \\
POD CATH & & 5.8 & \\
D $/ C$ & & & \\
\hline
\end{tabular}

Note. Age in years; BSA, body surface area in M2; EBL, estimated blood loss in $\mathrm{ml}$; narcotic usage, mean per day of morphine sulfate equivalents; duration of surgery, mean hours; bowel function return on postoperative day; POD CATH D/C, post-op day catheter removed.

terectomy. The incidence of serious complications has continued to decrease. Several authors have recommended long-term bladder drainage as an adjunct to avoid lower tract complications [2-5]. In 1962 Green et al. reported a fistula rate of approximately $10 \%$ [2]. On the basis of their review, "it is clearly apparent" that prolonged vesical drainage reduced the incidence of fistulae and other lower tract abnormalities. Although current fistulae rates are less than $2 \%$ [8], it is unclear whether long-term indwelling catheters have contributed to this improvement.

Prolonged catheterization may contribute to bladder dysfunction. Long-term catheterization prevents the bladder from expanding and emptying in its usual fashion and acts as a chronic irritant, possibly contributing to vesical pathology. Conversely, intermittent self-catheterization "achieves a state relatively free of catheter and permits bladder filling to act as a stimulus for return of reflex bladder contractility" [16].

Several studies have documented vesical abnormalitics in patients following radical hysterectomy. Green et al. reported that $13 \%$ of patients experience temporary voiding difficulties of less than 6-month duration [2]. Carenza et al. demonstrated that patients had decreased functional bladder capacity, hypertonias, and abnormal bladder sensation following radical hysterectomy [5]. They recommended that continuous bladder drainage be routinely employed following radical hysterectomy. Forney demonstrated that patients had increased postvoid residuals and decreased sensation for an extended period following radical hysterectomy [4]. He correlated the duration of increased PVRs with the extent of cardinal ligament resection and found that residuals remained high longer in patients with completely resected ligaments. Long-term catheterization was recommended to avoid these problems. Similarly, Farquharson et al. found that postoper- ative radical hysterectomy patients experienced decreased bladder compliance in addition to decreased bladder sensation and retention [10]. They postulated that the degree of vesical dysfunction was related to the "radicality" of the procedure and that autonomic innervation via the cardinal and uterosacral ligaments was interrupted during the dissection and advocated long-term catheterization. In their study of 22 patients, mean duration of suprapubic catheters was 31 days. Seski and Diokno also felt that parasympathetic innervation via the hypogastric plexus coursing through the cardinal ligament was disrupted during radical surgery and that vesical dysfunction followed [7]. Their data were further supported by Kadar et al., who postulated that the level at which the cardinal ligaments were divided had a dramatic influence on the frequency, type, and severity of urinary problems that followed surgery [3]. Scotti et al. demonstrated similar postoperative dysfunction in 12 patients. Additionally, they showed that by 3 months no significant difference in first sensation, fullness sensation, maximum capacity, voiding patterns, residual volumes, or flow rates existed compared to those at preoperative evaluation [6]. To our knowledge it has not been demonstrated that early removal increases the incidence or severity of bladder complications following radical hysterectomy.

Several authors have reported that the bladder is innervated via autonomic fibers coursing through the cardinal and uterosacral ligaments [12-15]. These pathways are disrupted during radical hysterectomy and it would be reasonable to postulate that bladder function would be severely altered in all patients. As stated by Twombly, "the difficult thing to understand is why all patients do not have complete bladder denervation" [15]. However, this does not appear to be the case, since most patients eventually recover complete vesical function. Kadar $e t a l$. postulated that dysfunction can largely be avoided by conserving the lateral portions of the pelvic ligaments [3]. In our study all patients had complete resection of the cardinal and uterosacral ligaments and only $12 \%$ experienced significant dysfunction for longer than 8 weeks following surgery. All patients were voiding without difficulty by 6 months. Other factors must play a role in vesical function. Increased intravesical pressure, with the valsalva maneuver and concurrent reflex vesical contraction, and personal motivation to successfully return to "normalcy" likely affect restoration of bladder function postoperatively.

In our experience, there was no increased rate of infection when catheters were removed in the early postoperative period. Nearly $70 \%$ of study patients required no form of bladder care except the employment of a strict voiding schedule after removal of their catheters. The median duration of continued ISC for PVR was no different than the indwelling catheter duration reported in 
previous studies and was shorter than that in our historical controls.

The number of patients requiring catheter support for more than 30 days after surgery was greater in our historical control group than in those needing ISC. Although the number of patients assessed by cystometrics in this series is small, no patient had decreased bladder compliance and all were successfully managed with ISC.

On the basis of these data, early removal of indwelling Foley catheters after radical hysterectomy with ISC for PVR based on the postvoid residual $12 \mathrm{hr}$ after catheter removal has become routine management at the University of Michigan Medical Center.

\section{REFERENCES}

1. Kelso, J., and Funnell, J. Radical Wertheim hysterectomy, Am. J. Obstet. Gynecol. 99, 106 (1967).

2. Green, T. H., Meigs, J. V., et al. Urologic complications of radical Wertheim hysterectomy: Incidence, etiology, management, and prevention, Obstet. Gynecol. 20, 293 (1962).

3. Kadar, N., Saliba, N., and Nelson, J. H. The frequency, causes and prevention of severe urinary dysfunction after radical hysterectomy, Br. J. Obstet. Gynaecol. 90, 858 (1983).

4. Forney, J. P. The effect of radical hysterectomy on bladder physiology, Am. J. Obstet. Gynecol. 138, 374 (1980).
5. Carenza, L., Nobili, F., and Giacobini, S. Voiding disorders after radical hysterectomy, Gynecol. Oncol. 13, 213 (1982).

6. Scotti, R. J., Bergman, A., Bhatia, N. N., and Ostergard, D. R. Urodynamic changes in urethrovesical function after radical hysterectomy, Obstet. Gynecol. 68, 111 (1986).

7. Seski, J. C., and Diokno, A. C. Bladder dysfunction after radical abdominal hysterectomy, Am. J. Obstet. Gynecol. 128, 643 (1977).

8. Morrow, C., and Townsend, D. Synopsis of gynecologic oncology, Wiley, New York, 3rd ed. (1987).

9. Knapp, R., and Berkowitz, R. Gynecologic oncology, MacMillan Co., New York (1986).

10. Farquharson, D. I., Shingleton, H. M., and Orr, J. W. The shortterm effect of radical hysterectomy on urethral and bladder function, Br. J. Obstet. Gynaecol. 94, 351 (1987).

11. Piver, M., Rutledge, F., and Smith, J. Five classes of extended hysterectomy for women with cervical cancer, Obstet. Gynecol. 44, 265 (1974).

12. McGuire, E. J., and Savastano, J. A. Long-term followup of spinal cord injury patients managed by intermittent catheterization, $J$. Urol. 129, 775 (1983).

13. Mundy, A. R. An anatomical explanation for bladder dysfunction following rectal and uterine surgery, Br. J. Urol. 54, 501 (1982).

14. Smith, P. H., and Ballantyne, B. The neuroanatomical basis for denervation of the urinary bladder following major pelvic surgery, Br. J. Surg. 55, 62 (1968).

15. Twombly, G. D. The technique of radical hysterectomy for carcinoma of the cervix. Cancer 3, 975 (1950).

16. McGuire, E. J. The innervation and function of the lower urinary tract, J. Neurosurg. 65, 278 (1986). 